Pacific

Journal of

Mathematics

\title{
CONVERGENCE OF AXIALLY SYMMETRIC VOLUME-PRESERVING MEAN CURVATURE FLOW
}

MaRia ATHANASSENAS AND SEVVANDi KandanaARACHCHI 


\title{
CONVERGENCE OF AXIALLY SYMMETRIC VOLUME-PRESERVING MEAN CURVATURE FLOW
}

\author{
Maria ATHANASSENAS AND SEVVANDI KANDANAARACHCHI
}

\begin{abstract}
We study the convergence of axially symmetric hypersurfaces evolving by volume-preserving mean curvature flow. Assuming the surfaces do not develop singularities along the axis of rotation at any time during the flow, and without any additional conditions, as for example on the curvature, we prove that the flow converges to a hemisphere, when the initial hypersurface has a free boundary and satisfies Neumann boundary data, and to a sphere when it is compact without boundary.
\end{abstract}

\section{Introduction}

Consider $n$-dimensional hypersurfaces $M_{t}$, defined by a one-parameter family of smooth immersions $\boldsymbol{x}_{t}: M^{n} \rightarrow \mathbb{R}^{n+1}$. The hypersurfaces $M_{t}$ are said to move by mean curvature if $\boldsymbol{x}_{t}=\boldsymbol{x}(\cdot, t)$ satisfies

$$
\frac{d}{d t} \boldsymbol{x}(p, t)=-H(p, t) v(p, t), \quad p \in M^{n}, t>0,
$$

where $v(p, t)$ denotes a smooth choice of unit normal on $M_{t}$ at $\boldsymbol{x}(p, t)$ (outer normal in case of compact surfaces without boundary), and $H(p, t)$ the mean curvature with respect to this normal.

If in addition the evolving compact surfaces $M_{t}$ are assumed to enclose a prescribed volume $V$, the corresponding evolution equation is

$$
\frac{d}{d t} x(p, t)=-(H(p, t)-h(t)) v(p, t), \quad p \in M^{n}, t>0,
$$

where $h(t)$ is the average of the mean curvature,

$$
h(t)=\frac{\int_{M_{t}} H d g_{t}}{\int_{M_{t}} d g_{t}},
$$

and $g_{t}$ denotes the metric on $M_{t}$. As under the flow (1-1), the surface area $\left|M_{t}\right|$ of the hypersurface is known to decrease under (1-2), while the enclosed volume remains constant in the latter; see [Athanassenas 1997].

MSC2010: primary 53C44; secondary 35K93.

Keywords: volume-preserving mean curvature flow, mean curvature flow with constraints. 
We are interested in an axially symmetric surface, enclosing a given volume $V$, and which has a nonempty boundary contained in a plane $\Pi$ that is perpendicular to the axis of rotation. Motivated by the fact that the stationary solution to the associated variational problem satisfies a Neumann boundary condition, we also assume the surface to meet that plane $\Pi$ at right angles along its boundary. Assuming the surface to be smooth, it will also intersect the axis of rotation orthogonally.

We consider the case where the evolving hypersurfaces do not develop singularities, in particular they do not pinch off along the axis of rotation during the flow, having only one intersection with that axis at the point that is the furthest from the supporting plane $\Pi$, and prove that the flow converges to a half-sphere.

The methods we use apply also in the case of evolving axially symmetric hypersurfaces without boundary having a similar lower height bound, and in that case we prove in Section 8 that the flow converges to a sphere.

The results in this paper make use of the axial symmetry, but no additional conditions on the curvature of the surface are assumed. Convergence to spheres has been previously proved for the volume flow in [Huisken 1987], for compact, uniformly convex initial surfaces, while Li [2009] assumes bounds on the traceless second fundamental form.

Our results can be seen as complementing the work in [Athanassenas 1997; 2003], and in the PhD dissertation [Kandanaarachchi 2011]: in the case of the surface behaving like a "bridge" between two parallel surfaces, if one were able to flow through singularities, the axially symmetric volume-preserving flow would converge to a number of spheres and (possibly) two hemispheres on the parallel planes, like beads strung along the axis of rotation.

\section{Notation, definitions and assumptions}

In the case of the surface $M_{t}$ intersecting the obstacle $\Pi$, we will at different stages divide it into two parts as in [Altschuler et al. 1995]: one adjacent to the plane and one containing the (only) intersection with the axis of rotation.

Let $\Pi=\left\{\left(x_{1}, \ldots, x_{n+1}\right) \in \mathbb{R}^{n+1}: x_{1}=0\right\}$ and let $M_{t}$ be contained in the right half-space, $M_{t} \subset\left\{x_{1}>0\right\}$. We use $R_{t}$ as the generic notation for the part of the surface closest to the plane, and $C_{t}$ for the rest - the cap that intersects the axis of rotation - and we will introduce various superscripts depending on the situation that will be made clear in the text.

We denote by $P(t)=(d(t), 0)$ the pole: the point of intersection of $M_{t}$ with the axis of rotation. We assume that there are no singularities developing, so that $P(t)$ is the only point of intersection of $M_{t}$ with the axis of rotation for all time. We are interested in those solutions where the generating curve of the initial hypersurface is smooth and can be written as a graph over the $x_{1}$ axis except at the pole. 
We use the notation

$$
\rho_{t}:[0, d(t)] \rightarrow \mathbb{R}
$$

for the radius function of the surface of revolution.

Let $\boldsymbol{i}_{1}, \ldots, \boldsymbol{i}_{n+1}$ be the standard basis in $\mathbb{R}^{n+1}$ and let $\boldsymbol{i}_{1}$ be the direction of the axis of rotation. We denote the quantities associated with the cap with a tilde ${ }^{\sim}$, and in this context we work with the vertical graph equation [Altschuler et al. 1995].

Furthermore we define the following quantities on $M_{t}$ :

Let $\omega=\hat{\boldsymbol{x}} /|\hat{\boldsymbol{x}}| \in \mathbb{R}^{n+1}, \hat{\boldsymbol{x}}=\left(0, x_{2}, \ldots, x_{n+1}\right)$, denote the outer unit normal to the cylinder intersecting $M_{t}$ at the point $\boldsymbol{x}(p, t)$. We call $u=\langle\boldsymbol{x}, \omega\rangle$ the height function of $M_{t}$, and set $v=\langle v, \omega\rangle^{-1}$. Note that $v$ corresponds to $\sqrt{1+\dot{\rho}^{2}}$; it will be used to obtain gradient estimates.

The corresponding quantities on the cap $C_{t}$ are the height measured from the plane $\Pi, \tilde{u}=\left\langle\boldsymbol{x}, \boldsymbol{i}_{1}\right\rangle$ and $\tilde{v}=\left\langle\nu, \boldsymbol{i}_{1}\right\rangle^{-1}$.

We divide the hypersurface into two regions using a plane $L_{\alpha}(t)$, which is parallel to $\Pi$ and intersects the surface at points where $\left\langle v, \boldsymbol{i}_{1}\right\rangle_{\mid L_{\alpha}(t) \cap M_{t}}=1 / \alpha$, with $\alpha>1$ being a constant. We define the cap, determined by the inclination angle, as the connected component of $M_{t}$ containing the pole $P$,

$$
C_{t}^{\alpha}=\left\{\boldsymbol{x}(p, t) \in M_{t}: 1 / \alpha<\left\langle\nu, \boldsymbol{i}_{1}\right\rangle \leq 1\right\},
$$

and we call $R_{t}^{\alpha}=M_{t} \backslash C_{t}^{\alpha}$ the cylindrical part of the surface. Note that $L_{\alpha}(t)$ is chosen such that the specific inclination angle is achieved nowhere else between that plane and the pole $P(t)$. As long as the flow is smooth, $C_{t}^{\alpha}$ is by definition a graph over the $x_{1}$ axis except at the pole. We denote by $l_{\alpha}(t)$ the $x_{1}$ coordinate of $L_{\alpha}(t)$, so $L_{\alpha}(t)=\left\{x_{1}=l_{\alpha}(t)\right\}$.

Assumption 2.1. We assume that for any $\alpha>1$ there exists a constant $c(\alpha)>0$, depending only on $\alpha$, such that $u_{\mid R_{t}^{\alpha}}>c(\alpha)$, that is, we assume a lower height bound in $R_{t}^{\alpha}$, independent of time, dependent on $\alpha$.

Thus $P(t)$ is assumed to be the only point of intersection of $M_{t}$ with the axis of rotation for all time. The assumption prevents singularities from developing on the axis of rotation.

For an axially symmetric surface the mean curvature is given by

$$
H=-\frac{\ddot{\rho}}{\left(1+\dot{\rho}^{2}\right)^{3 / 2}}+\frac{n-1}{\rho\left(1+\dot{\rho}^{2}\right)^{1 / 2}},
$$

while the principal curvatures are $k=-\ddot{\rho} /\left(1+\dot{\rho}^{2}\right)^{3 / 2}$ and $p=1 /\left(\rho \sqrt{1+\dot{\rho}^{2}}\right)$.

We also introduce another quantity, $q=\left\langle\nu, \boldsymbol{i}_{1}\right\rangle u^{-1}$; thus $p^{2}+q^{2}=u^{-2}$. 


\section{Height estimates}

In this section we prove that $M_{t}$ satisfies uniform height bounds: both the height function $u$ defined above and the height when measured as distance from the obstacle $\Pi$, denoted by $\tilde{u}$, are bounded. That is then used to show that the length of the generating curve of the surface remains bounded.

Lemma 3.1. The evolving surfaces $M_{t}$ satisfy the uniform height bound

$$
u \leq R=\left(\left|M_{0}\right| / \omega_{n}\right)^{1 / n} .
$$

Proof. We follow a method used in [Athanassenas 1997] to get bounds for $u$. Given $R>0$, assume that $u_{M_{t}} \geq R$ at some given time $t$. Since the surface area is decreasing under the flow, and by comparing to the projection of the surface onto the plane $\Pi$, we have

$$
\left|M_{0}\right| \geq\left|M_{t}\right|>\omega_{n} R^{n},
$$

where $\omega_{n}$ is the volume of the $n$ dimensional unit ball. Therefore $R>\left(\left|M_{0}\right| / \omega_{n}\right)^{1 / n}$ would contradict the fact that the evolution decreases the surface area.

Lemma 3.2. There is a constant $l$ such that the evolving surfaces $M_{t}$ satisfy the height bound $\tilde{u} \leq l$, that is, the distance from the plane $\Pi$ is uniformly bounded. Proof. Here $\alpha=1 / \cos \theta$,

$$
C_{t}^{\alpha}=\left\{\boldsymbol{x}(p, t) \in M_{t}: 1 / \alpha<\left\langle v, \boldsymbol{i}_{1}\right\rangle \leq 1\right\},
$$

and $R_{t}^{\alpha}=M_{t} \backslash C_{t}^{\alpha}$. From Assumption 2.1, we know that $u>c(\alpha)$ in $R_{t}^{\alpha}$. As $u_{\mid \partial C_{t}^{\alpha}} \leq R$ by Lemma 3.1 and $|\dot{\rho}| \geq \tan \left(\frac{\pi}{2}-\theta\right)=1 / \sqrt{\alpha^{2}-1}$ in $C_{t}^{\alpha}$, we have

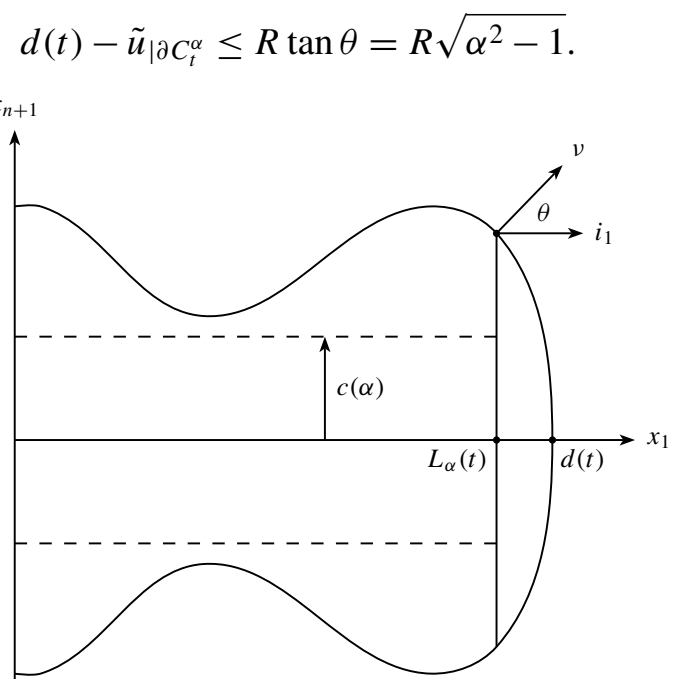

Figure 1. The cylinder of radius $c(\alpha)$. 
Assume there exists a length $l_{1}$ such that $\tilde{u}_{\mid R_{t}^{\alpha}}>l_{1}$. Then

$$
\left|M_{0}\right| \geq\left|M_{t}\right|>n \omega_{n} c^{n-1}(\alpha) l_{1},
$$

where now we compared $\left|M_{t}\right|$ to the surface area of an $n$ dimensional cylinder of radius $c(\alpha)$ and length $l_{1}$. Having

$$
l_{1}>\frac{\left|M_{0}\right|}{n \omega_{n} c^{n-1}(\alpha)}
$$

would contradict the fact that the evolution decreases the surface area. Therefore

$$
\tilde{u}<\frac{\left|M_{0}\right|}{n \omega_{n} c^{n-1}(\alpha)}+R \sqrt{\alpha^{2}-1}=: l .
$$

Next we show that the length of the generating curve is bounded.

Lemma 3.3. Assume $M_{t}$ to be smooth, axially symmetric hypersurfaces, evolving by (1-2) and with a radius function satisfying $\rho\left(x_{1}, t\right)>0$ for $x_{1} \in[0, d(t))$. Then there exists a constant $c_{*}$, independent of time, such that

$$
\int_{0}^{d(t)} \sqrt{1+\dot{\rho}^{2}} d x_{1} \leq c_{*}
$$

Proof. Let us divide $M_{t}$ into $R_{t}^{\alpha}$ and $C_{t}^{\alpha}$ for any $\alpha>1$. As the surface area is decreasing under the flow, $\left|M_{t}\right| \leq\left|M_{0}\right|$, we have

$$
\begin{gathered}
2 \pi \int_{0}^{d(t)} \rho^{n-1} \sqrt{1+\dot{\rho}^{2}} d x_{1} \leq\left|M_{0}\right|, \\
2 \pi \int_{0}^{l_{\alpha}(t)} \rho^{n-1} \sqrt{1+\dot{\rho}^{2}} d x_{1} \leq 2 \pi \int_{0}^{d(t)} \rho^{n-1} \sqrt{1+\dot{\rho}^{2}} d x_{1} \leq\left|M_{0}\right| .
\end{gathered}
$$

From Assumption 2.1,

$$
2 \pi c^{n-1}(\alpha) \int_{0}^{l_{\alpha}(t)} \sqrt{1+\dot{\rho}^{2}} d x_{1} \leq\left|M_{0}\right| \quad \text { and } \quad \int_{0}^{l_{\alpha}(t)} \sqrt{1+\dot{\rho}^{2}} d x_{1} \leq \frac{\left|M_{0}\right|}{2 \pi c^{n-1}(\alpha)} .
$$

We can estimate the length of the generating curve of the cap $C_{t}^{\alpha}$ by $l+R$. Therefore

$$
\int_{0}^{d(t)} \sqrt{1+\dot{\rho}^{2}} d x_{1} \leq \frac{\left|M_{0}\right|}{2 \pi c^{n-1}(\alpha)}+l+R=: c_{*} .
$$

\section{Estimates on $h$}

We now derive a priori estimates for $h(t)$ for solutions of the graphical equation.

Lemma 4.1. Assume $M_{t}$ to be smooth, axially symmetric hypersurfaces, evolving by (1-2) and with a radius function satisfying $\rho\left(x_{1}, t\right)>0$ for $x_{1} \in[0, d(t))$. Then there is a constant $c_{1}$ such that $0 \leq h(t) \leq c_{1}$ throughout the flow. 
Proof. Following [Athanassenas 2003] we parametrize $M_{t}$ by its radius function $\rho \in C^{\infty}([0, d(t)))$, then clearly

$$
H=-\frac{\ddot{\rho}}{\left(1+\dot{\rho}^{2}\right)^{3 / 2}}+\frac{n-1}{\rho\left(1+\dot{\rho}^{2}\right)^{1 / 2}} .
$$

From Lemma 3.3, we know that $\int_{0}^{d(t)} \sqrt{1+\dot{\rho}^{2}} d x_{1} \leq c_{*}$. Our proof follows the ideas of [Athanassenas 1997], the difference being the boundary term when integrating by parts. For the sake of completeness we include it here. For the second term of

$$
h(t)=\frac{1}{\left|M_{t}\right|} \int_{M_{t}}(k+(n-1) p) d g_{t}, \quad t \in[0, T),
$$

we have

$$
0 \leq \frac{n-1}{\left|M_{t}\right|} \int_{0}^{d(t)} \rho^{n-2}\left(x_{1}, t\right) d x_{1} \leq \frac{(n-1) R^{n-2} l}{\left|M_{t}\right|},
$$

since $\rho \leq R$ and $d(t) \leq l$ by Lemmas 3.1 and 3.2.

For the first term note that $\ddot{\rho} /\left(1+\dot{\rho}^{2}\right)=\frac{d}{d x_{1}}(\arctan \dot{\rho})$. Therefore

$$
\begin{aligned}
\int_{M_{t}} k d g_{t} & =-\int_{0}^{d(t)} \frac{d}{d x_{1}}(\arctan \dot{\rho}) \rho^{n-1} d x_{1} \\
& =\left.(\arctan \dot{\rho}) \rho^{n-1}\right|_{x_{1}=0}-\left.(\arctan \dot{\rho}) \rho^{n-1}\right|_{x_{1}=d(t)} \\
& \quad+(n-1) \int_{0}^{d(t)}(\arctan \dot{\rho}) \dot{\rho} \rho^{n-2} d x_{1} \\
& =(n-1) \int_{0}^{d(t)}(\arctan \dot{\rho}) \dot{\rho} \rho^{n-2} d x_{1},
\end{aligned}
$$

because $\arctan \dot{\rho}=0$ when $x_{1}=0$, and we have $\rho(d(t))=0$ at the pole. Since $0 \leq(\arctan \dot{\rho}) \dot{\rho} \leq \frac{\pi}{2}|\dot{\rho}| \leq \frac{\pi}{2} \sqrt{1+\dot{\rho}^{2}}$, we obtain

$$
\begin{aligned}
0 \leq \frac{1}{\left|M_{t}\right|} \int_{M_{t}} k d g_{t} & \leq \frac{(n-1)}{\left|M_{t}\right|} \frac{\pi}{2} \int_{0}^{d(t)} \sqrt{1+\dot{\rho}^{2}} \rho^{n-2} d x_{1} \\
& \leq \frac{(n-1) R^{n-2}}{\left|M_{t}\right|} \frac{\pi}{2} \int_{0}^{d(t)} \sqrt{1+\dot{\rho}^{2}} d x_{1} \leq \frac{(n-1) c_{*} R^{n-2}}{\left|M_{t}\right|} \frac{\pi}{2}
\end{aligned}
$$

where we have used Lemma 3.3.

From the isoperimetric inequality and the fact that the flow decreases surface area we know that

$$
V^{n /(n+1)}<c\left|M_{t}\right| \leq c\left|M_{0}\right|
$$

Combining these arguments we conclude that $0 \leq \frac{\int H d g}{\int d g} \leq c_{1}$. 


\section{Evolution equations and gradient estimates}

The maximum principle for noncylindrical or time dependent domains is discussed in [Lumer and Schnaubelt 1999]. We use that version of the maximum principle in this paper.

Lemma 5.1. For the flow (1-2) we have the following evolution equations:

(i) $\left(\frac{d}{d t}-\Delta\right) u=h / v-(n-1) / u$.

(ii) $\left(\frac{d}{d t}-\Delta\right) \tilde{u}=h / \tilde{v}$.

(iii) $\left(\frac{d}{d t}-\Delta\right) v=-|A|^{2} v+(n-1) v / u^{2}-(2 / v)|\nabla v|^{2}$.

(iv) $\left(\frac{d}{d t}-\Delta\right) \tilde{v}=-|A|^{2} \tilde{v}-(2 / \tilde{v})|\nabla \tilde{v}|^{2}$.

(v) $\left(\frac{d}{d t}-\Delta\right) H=(H-h)|A|^{2}$.

(vi) $\left(\frac{d}{d t}-\Delta\right)|A|^{2}=-2|\nabla A|^{2}+2|A|^{4}-2 h C$.

(vii) $\left(\frac{d}{d t}-\Delta\right) p=|A|^{2} p+2 q^{2}(k-p)-h p^{2}$.

(viii) $\left(\frac{d}{d t}-\Delta\right) k=|A|^{2} k-2(n-1) q^{2}(k-p)-h k^{2}$.

where $C=g^{i j} g^{k l} g^{m n} h_{i k} h_{l m} h_{n j}$, with $g^{i j}$ denoting the components of the inverse of the first fundamental form, and $h_{i j}$ those of the second fundamental form.

Proof. (i) and (iii) are proved in [Athanassenas 1997]; (v) and (vi) in [Huisken 1987].

(ii) For $\tilde{u}=\left\langle\boldsymbol{x}, \boldsymbol{i}_{1}\right\rangle$ we have

$$
\frac{d}{d t} \tilde{u}=\left\langle\frac{d}{d t} \boldsymbol{x}, \boldsymbol{i}_{1}\right\rangle=-(H-h)\left\langle v, \boldsymbol{i}_{1}\right\rangle \quad \text { and } \quad \Delta \tilde{u}=\left\langle\Delta \boldsymbol{x}, \boldsymbol{i}_{1}\right\rangle=-H\left\langle v, \boldsymbol{i}_{1}\right\rangle,
$$

so that

$$
\left(\frac{d}{d t}-\Delta\right) \tilde{u}=h\left\langle v, i_{1}\right\rangle
$$

(iv) For $\tilde{v}=\left\langle v, \boldsymbol{i}_{1}\right\rangle^{-1}$ we have

$$
\frac{d}{d t} \tilde{v}=-\tilde{v}^{2}\left\langle\frac{d}{d t} v, \boldsymbol{i}_{1}\right\rangle=-\tilde{v}^{2}\left\langle\nabla H, \boldsymbol{i}_{1}\right\rangle .
$$

The evolution equation follows from the well-known identity [Ecker and Huisken 1989]

$$
\Delta \tilde{v}=-\tilde{v}^{2}\left\langle\nabla H, i_{1}\right\rangle+\tilde{v}|A|^{2}+2 \tilde{v}^{-1} \nabla \tilde{v}^{2} .
$$

(vii) Using the same approach as in [Huisken 1990], we start with

$$
\begin{aligned}
\frac{d}{d t} p= & \frac{d}{d t}\left(u^{-2}-q^{2}\right)^{1 / 2} \\
=\Delta p+ & p^{-1}|\nabla p|^{2}+p^{-1}|\nabla q|^{2}-3 p^{-1} u^{-4}|\nabla u|^{2}+p^{-1} u^{-4} \\
& \quad-q p^{-1}\left(|A|^{2} q+q\left(p^{2}-q^{2}-2 k p\right)\right)-h u^{-2}+h q^{2} .
\end{aligned}
$$


Equation (vii) follows then from the relations

$$
\nabla_{i} u=\delta_{i 1} q u, \quad \nabla_{1}\left\langle v, \boldsymbol{i}_{1}\right\rangle=k p u, \quad \nabla_{i} q=\delta_{i 1}\left(q^{2}+k p\right),
$$

$$
\nabla_{i} p=\delta_{i 1} q(p-k), \quad|A|^{2}=k^{2}+(n-1) p^{2}, \quad u^{-4}=p^{4}+2 p^{2} q^{2}+q^{4} .
$$

(viii) The evolution equation for $H$ was derived in [Huisken 1987], and (viii) follows from (v), (vii), and the fact that $H=k+(n-1) p$.

We proceed to obtain gradient estimates in the different parts of the surface: for the cap by using the vertical graph equation and part (iv) from Lemma 5.1 above, and for the cylindrical part away from the cap by using the evolution equation (iii) in Lemma 5.1.

The quantities $\tilde{u}$ and $\tilde{v}$ are used on the cap.

Lemma 5.2. Assume $M_{t}$ to be axially symmetric surfaces as described in Section 2 that evolve by (1-2). Then the gradient estimate $\tilde{v} \leq \alpha$ holds on the cap $C_{t}^{\alpha}$. In addition, there is a constant $c_{2}(\alpha)$ such that $v \leq c_{2}(\alpha)$ for the cylindrical part $R_{t}^{\alpha}$.

Proof. Note that

$$
\left(\frac{d}{d t}-\Delta\right) \tilde{v} \leq 0
$$

so that by the maximum principle $\tilde{v} \leq \max \left(\max _{C_{0}^{\alpha}} \tilde{v}, \max _{\partial C_{t}^{\alpha}} \tilde{v}\right)$. By definition in $C_{t}^{\alpha}$ we have $\tilde{v} \leq \alpha$, and this is supported by the evolution equation!

From the assumption we know that $u>c(\alpha)$ in $R_{t}^{\alpha}$. As in [Athanassenas 1997, Proposition 4] we calculate

$$
\begin{aligned}
\left(\frac{d}{d t}-\Delta\right) u^{2} v & =-|A|^{2} u^{2} v+(n-1) v+2 u h-2(n-1) v-2 v|\nabla u|^{2}-\frac{2}{v} \nabla v \nabla\left(u^{2} v\right) \\
& \leq 2 h u-(n-1) v .
\end{aligned}
$$

If $v>2 c_{1} R /(n-1)$ the right side is negative, and proceeding as in [Athanassenas 1997] we conclude $v \leq c_{2}(\alpha)$ in $R_{t}^{\alpha}$. It is important to note that on the boundary of $R_{t}^{\alpha}$, either $v=1$ (along the intersection with $\Pi$ ), or $v=\alpha / \sqrt{\alpha^{2}-1}$. Thereby, we have bounds for $v$ and $\tilde{v}$ in $R_{t}^{\alpha}$ and $C_{t}^{\alpha}$ respectively.

Remark 5.3. (i) The gradient bounds from Lemma 5.2 guarantee that $R_{t}^{\alpha}$ remains a graph. As $C_{t}^{\alpha}$ remains a graph for all $\alpha>1$, we see that $M_{t} \backslash P(t)$ remains a graph throughout the flow.

(ii) As the height of the graph is bounded we find a lower bound for the minimum $d(t)$ from

$$
V=\int_{0}^{d(t)} \omega_{n} \rho^{n}(x) d x_{1} \leq \omega_{n} R^{n} \int_{0}^{d(t)} d x_{1}=\omega_{n} R^{n} d(t) .
$$




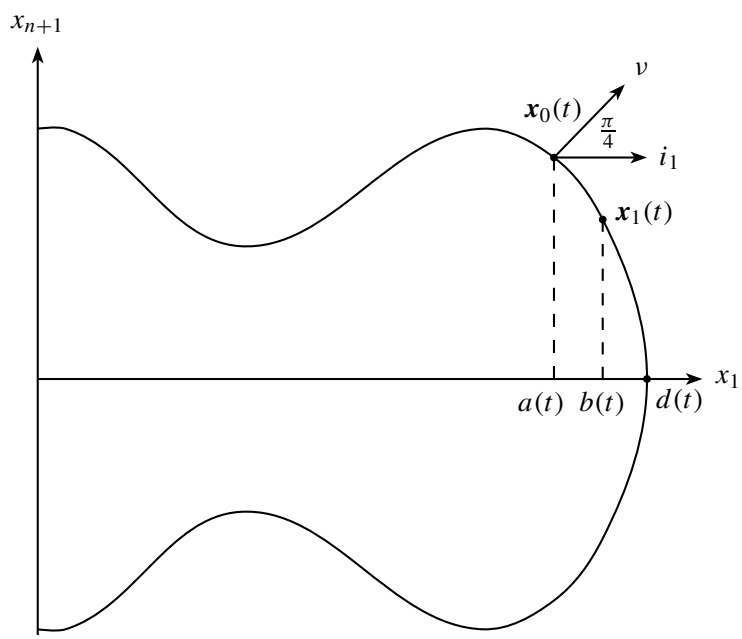

Figure 2. $x_{0}(t)$ - the boundary point of $C_{t}^{\sqrt{2}}$.

Lemma 5.4. Assume the $M_{t}$ to be axially symmetric surfaces as described in Section 2 and to evolve by (1-2). Let $\boldsymbol{x}_{0}(t)$ be a boundary point of $C_{t}^{\sqrt{2}}$, which we can assume without loss of generality to lie on the generating curve and be such that $\left\langle v\left(\boldsymbol{x}_{0}(t)\right), \boldsymbol{i}_{1}\right\rangle=1 / \sqrt{2}$ (with some abuse of notation for the corresponding normal $\left.v\left(x_{0}(t)\right)\right)$. Then $H\left(x_{0}(t)\right) \geq 0$ for $0 \leq t \leq T_{\max } \leq \infty$.

Proof. Suppose $H\left(x_{0}(t)\right)<0$, then by continuity there is a connected region $C_{t}^{\sqrt{2}, H^{-}} \subset C_{t}^{\sqrt{2}}$, with $x_{0}(t) \in \partial C_{t}^{\sqrt{2}, H^{-}}$, which clearly can be chosen to be axially

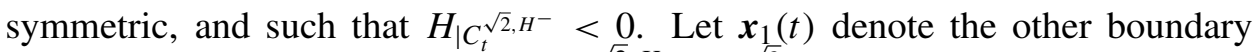
point along the generating curve in $C_{t}^{\sqrt{2}, H^{-}} \subset C_{t}^{\sqrt{2}}$, and let $a(t)=\left\langle x_{0}(t), \boldsymbol{i}_{1}\right\rangle$ and $b(t)=\left\langle\boldsymbol{x}_{1}(t), \boldsymbol{i}_{1}\right\rangle$ denote the $x_{1}$ coordinate of $\boldsymbol{x}_{0}(t), \boldsymbol{x}_{1}(t)$, respectively. Then

$$
0>\int_{C_{t}^{\sqrt{2}, H^{-}}} H d g=\int_{a(t)}^{b(t)}\left(-\frac{\ddot{\rho}}{1+\dot{\rho}^{2}} \rho^{n-1}+(n-1) \rho^{n-2}\right) d x_{1} .
$$

The second term being positive means that the first is negative, and given the bounds on the radius we find

$$
\int_{a(t)}^{b(t)}\left(-\frac{\ddot{\rho}}{1+\dot{\rho}^{2}}\right) d x_{1}=\int_{a(t)}^{b(t)}\left(-\frac{d}{d x_{1}}(\arctan \dot{\rho})\right) d x_{1}<0 .
$$

This results in

$$
\arctan \dot{\rho}(a(t))<\arctan \dot{\rho}(b(t)) \quad \text { and } \quad-\frac{\pi}{4}<\arctan \dot{\rho}(b(t)),
$$

by the choice of $a(t)$. But this is not possible in $C_{t}^{\sqrt{2}}$, where $-\frac{\pi}{2} \leq \arctan \dot{\rho}<-\frac{\pi}{4}$, contradicting our assumption and therefore $H\left(x_{0}(t)\right) \geq 0$. 


\section{Curvature estimates}

Proposition 6.1. Assume $M_{t}$ to be axially symmetric surfaces as described in Section 2 that evolve by (1-2). Then there is a constant $c_{2}$ depending only on the initial hypersurface, such that the principal curvatures $k$ and $p$ satisfy $k / p<c_{2}$, independently of time.

Proof. We calculate from Lemma 5.1 that

$$
\frac{d}{d t}\left(\frac{k}{p}\right)=\Delta \frac{k}{p}+\frac{2}{p} \nabla_{i} p \nabla_{i}\left(\frac{k}{p}\right)+2 \frac{q^{2}}{p^{2}}(p-k)((n-1) p+k)+\frac{h k}{p}(p-k) .
$$

If $k / p \geq 1$ then $(h k / p)(p-k)<0$. This implies that

$$
\frac{k}{p} \leq \max \left(1, \max _{M_{0}} \frac{k}{p}\right) \text {. }
$$

Note that for this consideration, the smooth function $k / p$ is defined over the whole surface, and in view of the orthogonality on the boundary, via a reflection argument there are no boundary data involved.

Proposition 6.2. Assume $M_{t}$ to be axially symmetric surfaces as described in Section 2 that evolve by (1-2) and let $A$ be the second fundamental form. Then there exists a constant $c_{3}$, independent of time, such that $|A|^{2} \leq c_{3}$.

Proof. We proceed as in [Ecker and Huisken 1991] and [Athanassenas 1997] and calculate the evolution equation for the product $g=|A|^{2} \varphi\left(v^{2}\right)$ in $R_{t}^{\sqrt{2}}$, where $\varphi(r)=r /(\lambda-\mu r)$, with $v=\langle\nu, \omega\rangle^{-1}$ and appropriately chosen constants $\lambda, \mu>0$. From the evolution equation of $g$ we find the inequality

$$
\begin{aligned}
& \left(\frac{d}{d t}-\Delta\right) g \\
& \quad \leq-2 \mu g^{2}-2 \lambda \varphi v^{-3} \nabla v \cdot \nabla g-\frac{2 \lambda \mu}{\left(\lambda-\mu v^{2}\right)^{2}}|\nabla v|^{2} g-2 h C \varphi\left(v^{2}\right)+\frac{2(n-1)}{u^{2}} v^{2} \varphi^{\prime}|A|^{2} .
\end{aligned}
$$

We estimate the second last term as in [Athanassenas 1997] using Young's inequality and obtain

$$
\begin{aligned}
-2 h C \varphi\left(v^{2}\right) & \leq 2 h|A|^{3} \varphi\left(v^{2}\right) \\
& \leq \frac{3}{2}|A|^{4} \varphi\left(v^{2}\right)+\frac{1}{2} h^{4} \varphi^{-2}\left(v^{2}\right)=\frac{3}{2} g^{2}+\frac{1}{2} h^{4} \varphi^{-2}\left(v^{2}\right) .
\end{aligned}
$$

We choose $\mu>\frac{3}{4}$ and $\lambda>\mu \max v^{2}$. As $\varphi^{\prime} v^{2}=\frac{\lambda}{\left(\lambda-\mu v^{2}\right)^{2}} \varphi$ we have

$$
\frac{2(n-1)}{u^{2}} v^{2} \varphi^{\prime}|A|^{2}=\frac{2(n-1) \lambda}{u^{2}\left(\lambda-\mu v^{2}\right)} g .
$$

As $u>c(1 / \sqrt{2})=c_{0}$ in $R_{t}^{\sqrt{2}}$ we get

$$
\frac{2(n-1) \lambda}{u^{2}\left(\lambda-\mu v^{2}\right)} g \leq c_{4} g .
$$


Therefore we have

$$
\begin{aligned}
\left(\frac{d}{d t}-\Delta\right) g & \leq-c_{5} g^{2}+c_{6} g-c_{7} \nabla v \cdot \nabla g+c_{8}(h, \max v) \\
& \leq-c_{5}\left(g-\frac{c_{6}}{2 c_{5}}\right)^{2}-c_{7} \nabla v \cdot \nabla g+c_{9} .
\end{aligned}
$$

The right side of this inequality is negative at a maximum of $g$, where

$$
g>\frac{c_{6}}{2 c_{5}}+\sqrt{\frac{c_{9}}{c_{5}}} .
$$

On $\partial R_{t}^{\sqrt{2}}$ we have $H=k+(n-1) p \geq 0$ by Lemma 5.4. Also, as $k / p<c_{2}$, we get $|k| / p<c$ on this boundary and thus we have

$$
|A|^{2}=k^{2}+(n-1) p^{2} \leq\left(c^{2}+n-1\right) p^{2} \leq C \rho^{-2} \leq C c_{0}^{-2}
$$

on $\partial R_{t}^{\sqrt{2}}$. By the maximum principle,

$$
g \leq \max \left(\max _{R_{0}^{\sqrt{2}}} g, \max _{\partial R_{t}^{\sqrt{2}}}|A|^{2} \varphi\left(v^{2}\right)\right) .
$$

Since $v \leq c_{2}(\sqrt{2})$ and $\varphi\left(v^{2}\right)$ is bounded, we have a bound for $g$ in $R_{t}^{\sqrt{2}}$.

The evolution equation for $\tilde{g}=|A|^{2} \varphi\left(\tilde{v}^{2}\right)$ on $C_{t}^{\sqrt{2}}$ is the same as the one for $g$ without the last term on the right side. Thereby we obtain a bound for $\tilde{g}$ in the same way as above.

Proposition 6.3. Assume $M_{t}$ to be axially symmetric surfaces as described in Section 2 that evolve by (1-2). Then for each $m \geq 1$ there is a $C_{m}$ such that

$$
\left|\nabla^{m} A\right|^{2} \leq C_{m},
$$

uniformly on $M_{t}$, for $0 \leq t \leq T_{\max } \leq \infty$.

Proof. Having obtained uniform bounds on $|A|^{2}$ and $h$ the proof is a repetition of that of Theorem 4.1 in [Huisken 1987].

Thus we have long-time existence for the flow:

Corollary 6.4. Let $M_{t}$ be axially symmetric surfaces as described in Section 2 that evolve by (1-2). Then

$$
T_{\max }=\infty
$$

\section{Convergence to surfaces of constant mean curvature}

Having long-time existence, Proposition 8 of [Athanassenas 1997] gives convergence to a constant mean curvature surface, which in our case is axially symmetric. By the classification of the Delaunay surfaces [1841] it has to be a half-sphere. 


\section{Other convergence results}

Using the same estimates with very few changes one can show that a compact, axially symmetric surface without boundary, which encloses a volume $V$ and intersects the axis only at two endpoints throughout the flow by (1-2), will converge to a sphere. We will only explain the parts that are different from the previous result.

We denote the surface again by $M_{t}$. We split it into a cylindrical part $R_{t}^{\alpha}$ and two caps $C_{t}^{(\alpha, i)}$, for $i=1,2$, in this case. The left side cap, $C_{t}^{(\alpha, 1)}$, intersects the axis of rotation at $x_{1}=e(t)$, while the (only other) intersection on the right for $C_{t}^{(\alpha, 2)}$ is located at $x_{1}=d(t)$. Assumption 2.1 holds on $M_{t}$.

Height estimates. The height estimates of Lemmas 3.1 and 3.2 change as follows:

Lemma 8.1. Assume $M_{t}$ to be axially symmetric, compact without boundary and evolving by (1-2). Then the height function $u$ satisfies $u<R=\left(\left|M_{0}\right| / 2 \omega_{n}\right)^{1 / n}$.

Proof. Given $R>0$ assume that $u_{M_{t}} \geq R$ at some given time $t$. Take a plane perpendicular to the $x_{1}$-axis and intersecting the surface. This plane divides the surface into two parts, and by projecting both parts onto it we find

$$
\left|M_{0}\right| \geq\left|M_{t}\right|>2 \omega_{n} R^{n} .
$$

Taking $R>\left(\frac{\left|M_{0}\right|}{2 \omega_{n}}\right)^{1 / n}$ would contradict the fact that the evolution decreases the surface area.

The next lemma gives an estimate for the diameter of $M_{t}$ in the $x_{1}$ direction.

Lemma 8.2. Assume $M_{t}$ to be smooth, axially symmetric, compact without boundary and evolving by (1-2). Then

$$
d(t)-e(t)<l=\frac{\left|M_{0}\right|}{n \omega_{n} c_{0}^{n-1}}+2 R .
$$

Proof. As in Lemma 3.2 let $\alpha=1 / \cos \theta$. From Assumption 2.1 we know that $u>c(\alpha)$ in $R_{t}^{\alpha}$. As $u_{\mid \partial C_{t}^{\alpha, i}} \leq R$ and $|\dot{\rho}| \geq \tan \left(\frac{\pi}{2}-\theta\right)$ in $C_{t}^{\alpha, i}$ for $i=1,2$, we have

$$
\begin{aligned}
& d(t)-\tilde{u}_{\mid \partial C_{t}^{\alpha, 1}} \leq R \tan \theta=R \sqrt{\alpha^{2}-1}, \\
& \tilde{u}_{\mid \partial C_{t}^{\alpha, 2}}-e(t) \leq R \tan \theta=R \sqrt{\alpha^{2}-1} .
\end{aligned}
$$

Assume there exists a length $l_{1}$ such that $\tilde{u}_{\mid R_{t}^{\alpha}}>l_{1}$. Then by the previous argument,

$$
\left|M_{0}\right| \geq\left|M_{t}\right|>n \omega_{n} c^{n-1}(\alpha) l_{1},
$$

where now we compared $\left|M_{t}\right|$ to the surface area of an $n$ dimensional cylinder of radius $c(\alpha)$ and length $l_{1}$. If $l_{1}>\left|M_{0}\right| /\left(n \omega_{n} c^{n-1}(\alpha)\right)$ this would contradict the fact 
that the evolution decreases the surface area. Therefore

$$
\tilde{u}<\frac{\left|M_{0}\right|}{n \omega_{n} c_{0}^{n-1}}+2 R \sqrt{\alpha^{2}-1} .
$$

Again we can estimate the length of the generating curve throughout the flow:

Lemma 8.3. Assume $M_{t}$ to be smooth, axially symmetric, compact without boundary, evolving by (1-2) and with a radius function satisfying $\rho\left(x_{1}, t\right)>0$ for $x_{1}$ in $(e(t), d(t))$. Then there exists a constant $c_{*}$, independent of time, such that

$$
\int_{0}^{d(t)} \sqrt{1+\dot{\rho}^{2}} d x_{1} \leq c_{*}
$$

Proof. The proof is the same as that of Lemma 3.3 after taking into account the two caps on either side. Here we have

$$
\int_{e(t)}^{d(t)} \sqrt{1+\dot{\rho}^{2}} d x_{1} \leq \frac{\left|M_{0}\right|}{2 \pi c^{n-1}(\alpha)}+2 l+2 R=: c_{*} .
$$

Lemma 8.4 (estimates on $h$ ). Assume $M_{t}$ to be smooth, axially symmetric, compact without boundary, evolving by (1-2) and with a radius function that satisfies $\rho\left(x_{1}, t\right)>0$ for $x_{1} \in(e(t), d(t))$. Then there is a constant $c_{1}$ such that $0 \leq h(t) \leq c_{1}$ throughout the flow.

Proof. The only change to the proof of Lemma 4.1 is in the boundary values when integrating by parts in (4-1). Here the new boundary values are

$$
\left.(\arctan \dot{\rho}) \rho^{n-1}\right|_{x_{1}=a(t)}-\left.(\arctan \dot{\rho}) \rho^{n-1}\right|_{x_{1}=b(t)} .
$$

As $\rho(a(t))=\rho(b(t))=0$, the boundary terms disappear and we get the same estimate for $h$.

Lemma 8.5 (gradient estimates). Under the above assumptions, the gradient estimate $|\tilde{v}| \leq \alpha$ holds on the caps $C_{t}^{\alpha, i}, i=1,2$. In addition there is a constant $c$, such that $v \leq c$ for the cylindrical part $R_{t}^{\alpha}$.

Proof. The gradient estimates are as in Lemma 5.2, but in this setting instead of one cap $C_{t}^{\alpha}$ we have two caps on either side, and the same estimate holds for both caps.

Concluding this section, we remark that $H \geq 0$ at points where the caps $C_{t}^{\sqrt{2}, i}$, $i=1,2$, meet the cylindrical part $R_{t}^{\sqrt{2}}$ of the surface. The proof is using the same arguments as the one for Lemma 5.4 after the appropriate adjustments of the sign of $\arctan \dot{\rho}$ for the cap on the left of the surface. The results on curvature estimates and the convergence to a limiting surface of constant mean curvature follow along the same lines as previously proved. In this case the limit surface is a sphere. 


\section{References}

[Altschuler et al. 1995] S. Altschuler, S. B. Angenent, and Y. Giga, "Mean curvature flow through singularities for surfaces of rotation”, J. Geom. Anal. 5:3 (1995), 293-358. MR 97j:58029 Zbl 0847. 58072

[Athanassenas 1997] M. Athanassenas, "Volume-preserving mean curvature flow of rotationally symmetric surfaces", Comment. Math. Helv. 72:1 (1997), 52-66. MR 98d:58037 Zbl 0873.35033

[Athanassenas 2003] M. Athanassenas, "Behaviour of singularities of the rotationally symmetric, volume-preserving mean curvature flow”, Calc. Var. Partial Differential Equations 17:1 (2003), 1-16. MR 2004c:35006 Zbl 1045.53045

[Delaunay 1841] C. Delaunay, "Sur la surface de révolution dont la courbure moyenne est constante", J. Math. Pures Appl. 6 (1841), 309-320.

[Ecker and Huisken 1989] K. Ecker and G. Huisken, "Mean curvature evolution of entire graphs", Ann. of Math. (2) 130:3 (1989), 453-471. MR 91c:53006 Zbl 0696.53036

[Ecker and Huisken 1991] K. Ecker and G. Huisken, "Interior estimates for hypersurfaces moving by mean curvature", Invent. Math. 105:3 (1991), 547-569. MR 92i:53010 Zbl 0707.53008

[Huisken 1987] G. Huisken, "The volume preserving mean curvature flow", J. Reine Angew. Math. 382 (1987), 35-48. MR 89d:53015 Zbl 0621.53007

[Huisken 1990] G. Huisken, "Asymptotic behavior for singularities of the mean curvature flow", $J$. Differential Geom. 31:1 (1990), 285-299. MR 90m:53016 Zbl 0694.53005

[Kandanaarachchi 2011] S. Kandanaarachchi, Axially symmetric volume preserving mean curvature flow, thesis, Monash University, Melbourne, 2011.

[Li 2009] H. Li, "The volume-preserving mean curvature flow in Euclidean space", Pacific J. Math. 243:2 (2009), 331-355. MR 2010k:53100 Zbl 1182.53061

[Lumer and Schnaubelt 1999] G. Lumer and R. Schnaubelt, "Local operator methods and time dependent parabolic equations on non-cylindrical domains", pp. 58-130 in Evolution equations, Feshbach resonances, singular Hodge theory, edited by M. Demuth et al., Math. Top. 16, Wiley, Berlin, 1999. MR 2000j:35122 Zbl 0938.35067

Received September 13, 2011. Revised March 20, 2012.

MARIA ATHANASSENAS

SCHOOL OF MATHEMATICAL SCIENCES

MONASH UNIVERSITY

PO BOX 28M CLAYTON CAMPUS

MONASH UNIVERSITY VIC 3800

Australia

maria.athanassenas@sci.monash.edu.au

SEVVANDI KANDANAARACHCHI

SCHOOL OF MATHEMATICAL SCIENCES

MONASH UNIVERSITY

PO BOX 28M CLAYTON CAMPUS

MONASH UNIVERSITY VIC 3800

Australia

sevvandi.kandanaarachchi@monash.edu 


\title{
PACIFIC JOURNAL OF MATHEMATICS
}

\author{
http://pacificmath.org \\ Founded in 1951 by \\ E. F. Beckenbach (1906-1982) and F. Wolf (1904-1989)
}

\section{EDITORS}

V. S. Varadarajan (Managing Editor)

Department of Mathematics

University of California

Los Angeles, CA 90095-1555

pacific@math.ucla.edu

Vyjayanthi Chari

Department of Mathematics

University of California

Riverside, CA 92521-0135

chari@math.ucr.edu

\section{Robert Finn}

Department of Mathematics Stanford University

Stanford, CA 94305-2125

finn@math.stanford.edu

Kefeng Liu

Department of Mathematics

University of California

Los Angeles, CA 90095-1555

liu@math.ucla.edu
Darren Long

Department of Mathematics

University of California

Santa Barbara, CA 93106-3080

long@math.ucsb.edu

Jiang-Hua Lu

Department of Mathematics

The University of Hong Kong

Pokfulam Rd., Hong Kong jhlu@maths.hku.hk

Alexander Merkurjev

Department of Mathematics

University of California

Los Angeles, CA 90095-1555

merkurev@math.ucla.edu
Sorin Popa

Department of Mathematics University of California

Los Angeles, CA 90095-1555 popa@math.ucla.edu

Jie Qing

Department of Mathematics

University of California

Santa Cruz, CA 95064

qing@cats.ucsc.edu

Jonathan Rogawski

Department of Mathematics

University of California

Los Angeles, CA 90095-1555

jonr@math.ucla.edu

\section{PRODUCTION}

pacific@math.berkeley.edu

\section{SUPPORTING INSTITUTIONS}

ACADEMIA SINICA, TAIPEI

CALIFORNIA INST. OF TECHNOLOGY INST. DE MATEMÁTICA PURA E APLICADA KEIO UNIVERSITY

MATH. SCIENCES RESEARCH INSTITUTE NEW MEXICO STATE UNIV.

OREGON STATE UNIV.

\author{
STANFORD UNIVERSITY \\ UNIV. OF BRITISH COLUMBIA \\ UNIV. OF CALIFORNIA, BERKELEY \\ UNIV. OF CALIFORNIA, DAVIS \\ UNIV. OF CALIFORNIA, LOS ANGELES \\ UNIV. OF CALIFORNIA, RIVERSIDE \\ UNIV. OF CALIFORNIA, SAN DIEGO \\ UNIV. OF CALIF., SANTA BARBARA
}

\author{
UNIV. OF CALIF., SANTA CRUZ \\ UNIV. OF MONTANA \\ UNIV. OF OREGON \\ UNIV. OF SOUTHERN CALIFORNIA \\ UNIV. OF UTAH \\ UNIV. OF WASHINGTON \\ WASHINGTON STATE UNIVERSITY
}

These supporting institutions contribute to the cost of publication of this Journal, but they are not owners or publishers and have no responsibility for its contents or policies.

See inside back cover or pacificmath.org for submission instructions.

The subscription price for 2012 is US \$420/year for the electronic version, and \$485/year for print and electronic.

Subscriptions, requests for back issues from the last three years and changes of subscribers address should be sent to Pacific Journal of Mathematics, P.O. Box 4163, Berkeley, CA 94704-0163, U.S.A. Prior back issues are obtainable from Periodicals Service Company, 11 Main Street, Germantown, NY 12526-5635. The Pacific Journal of Mathematics is indexed by Mathematical Reviews, Zentralblatt MATH, PASCAL CNRS Index, Referativnyi Zhurnal, Current Mathematical Publications and the Science Citation Index.

The Pacific Journal of Mathematics (ISSN 0030-8730) at the University of California, c/o Department of Mathematics, 969 Evans Hall, Berkeley, CA 94720-3840, is published monthly except July and August. Periodical rate postage paid at Berkeley, CA 94704, and additional mailing offices. POSTMASTER: send address changes to Pacific Journal of Mathematics, P.O. Box 4163, Berkeley, CA 94704-0163.

PJM peer review and production are managed by EditFLOW ${ }^{\mathrm{TM}}$ from Mathematical Sciences Publishers.

PUBLISHED BY PACIFIC JOURNAL OF MATHEMATICS

at the University of California, Berkeley 94720-3840

A NON-PROFIT CORPORATION

Typeset in LATEX

Copyright $(02012$ by Pacific Journal of Mathematics 


\title{
PACIFIC JOURNAL OF MATHEMATICS
}

\author{
Volume $259 \quad$ No. $1 \quad$ September 2012
}

Extension Theorems for external cusps with minimal regularity

GABRIEL ACOSTA and IGNACIO OJEA

Convergence of axially symmetric volume-preserving mean curvature flow

MARIA ATHANASSENAS and SEVVANDi KANDANAARACHCHI

On the horoboundary and the geometry of rays of negatively curved manifolds

FranÇOISE DAL'bo, Marc PEIGNÉ and ANDREA

SAMBUSETTI

Two infinite versions of the nonlinear Dvoretzky theorem

KEI FUNANO

Nonlocal uniform algebras on three-manifolds

ALEXANDER J. IZZO

Mahlo cardinals and the torsion product of primary abelian groups

PATRICK W. KEEF

Geometry of trinomials

AARON MELMAN

Drinfeld orbifold algebras

ANNE V. SHEPLER and SARAH Witherspoon

Semi-topological cycle theory I

JYH-HAUR TEH

New construction of fundamental domains for certain Mostow groups 209

TiEHONG ZHAO 